\title{
Percutaneous coil embolization to manage pulmonary artery hemorrhage after distal endarterectomy
}

\author{
Chirantan Mangukia, MCh, ${ }^{\mathrm{a}}$ Paul Forfia, MD, ${ }^{\mathrm{b}}$ Anjali Vaidya, MD,${ }^{\mathrm{b}}$ Veronica Williams, DO, ${ }^{\mathrm{c}}$ \\ Dmitry Niman, MD, ${ }^{\mathrm{d}}$ Parth Rali, MD, ${ }^{\mathrm{e}}$ and Yoshiya Toyoda, MD, ${ }^{\mathrm{a}}$ Philadelphia, Pa
}

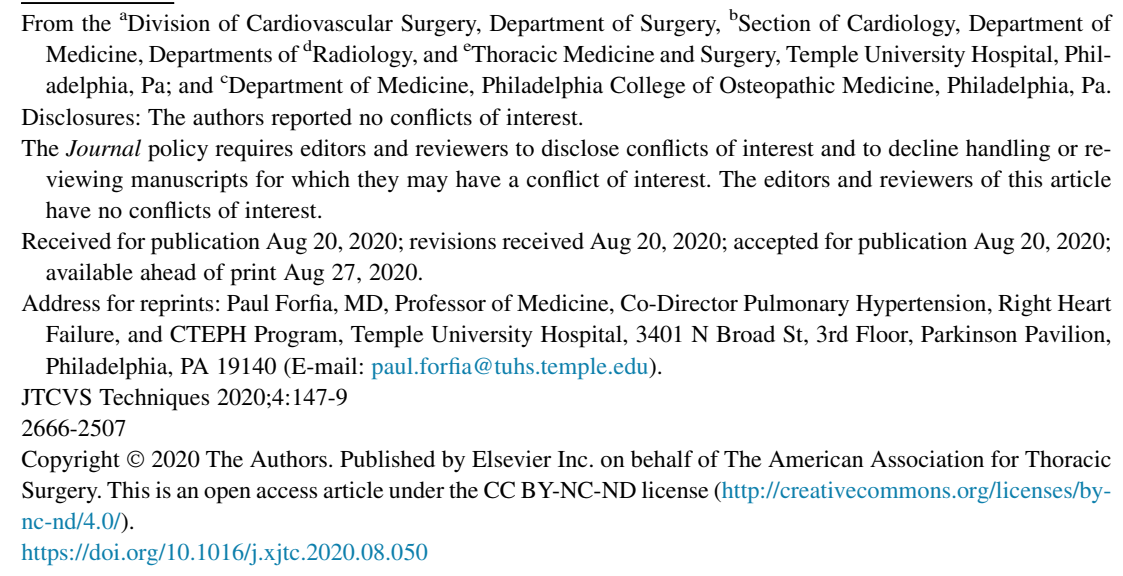

Video clip is available online.

A 68-year-old male patient with a medical history of diabetes mellitus, hypertension, and obstructive sleep apnea was referred to our pulmonary hypertension $(\mathrm{PH})$ program for chronic thromboembolic PH evaluation. Echocardiography revealed abnormal septal motion consistent with elevated right ventricular (RV) afterload, bi-atrial dilation, $\mathrm{RV}$ dilation with normal $\mathrm{RV}$ function, moderate $\mathrm{PH}$, and severe tricuspid regurgitation. Planar and single-photon emission computer tomography ventilation-perfusion scan showed perfusion defects predominantly in the right upper lobe and left lower lobe (LLL). Right heart catheterization showed severe $\mathrm{PH}$ with mean pulmonary artery (PA) pressure of $42 \mathrm{~mm} \mathrm{Hg}$ and pulmonary vascular resistance of 4.61 wood units $\left(368 \mathrm{dyn} \cdot \mathrm{s} \cdot \mathrm{cm}^{-5}\right)$. Pulmonary angiography revealed stenosis and perfusion defects in right upper lobe and complete occlusion of LLL artery (Figure 1). He was deemed a suitable candidate for pulmonary thromboendarterectomy (PTE) by the chronic thromboembolic PH multidisciplinary team.

Intraoperatively, the clot material was found to be severely adhered and partially calcified on the LLL branch (Figure 2). While being weaned off cardiopulmonary bypass (CPB), the patient had massive hemoptysis. He was placed back on CPB. Bronchoscopy showed significant bleeding from LLL. LLL endobronchial blocker (EBB) was placed to

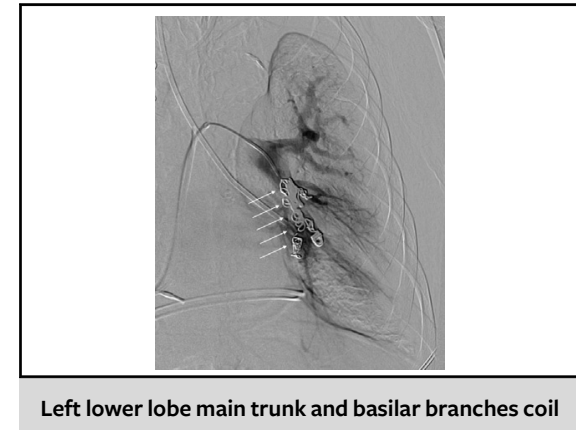

embolization.

CENTRAL MESSAGE

Selective pulmonary artery coil embolization can be a viable treatment option for acute pulmonary hemorrhage after pulmonary thromboendarterectomy.

See Commentaries on pages 150 and 152.

temporize bleeding into contralateral airways and peripheral veno-arterial extracorporeal membrane oxygenation (VAECMO) was used as a bridge to allow transition from CPB.

The patient was brought to the intensive care unit on VAECMO with a left-sided EBB in place in hemodynamically stable condition. The following day, under bronchoscopic guidance, EBB was deflated, and VA-ECMO flow was decreased from $4 \mathrm{~L} / \mathrm{min}$ to $2.5 \mathrm{~L} / \mathrm{min}$, allowing relative lung perfusion to assess for recurrent bleeding. Brisk bleeding was noted from the LLL, VA-ECMO flow was increased, and EBB reinflated. Recombinant activated Factor VII was injected into the LLL bronchus, but EBB deflation trial on day 2 showed continued bleeding.

Surgical lobectomy was considered as a last resort, as lobectomy may have to extend into left-sided pneumonectomy if the bleeding source is not localized in LLL. We thus pursued percutaneous coil embolization as the most viable and least-invasive approach, thinking that it would not result in LLL ischemia or infarction, given the patient's pre-PTE chronic vascular occlusion and related collateralization would be protective in this context. On day 2 , in the interventional radiology suite, EBB was deflated, ECMO flow was 


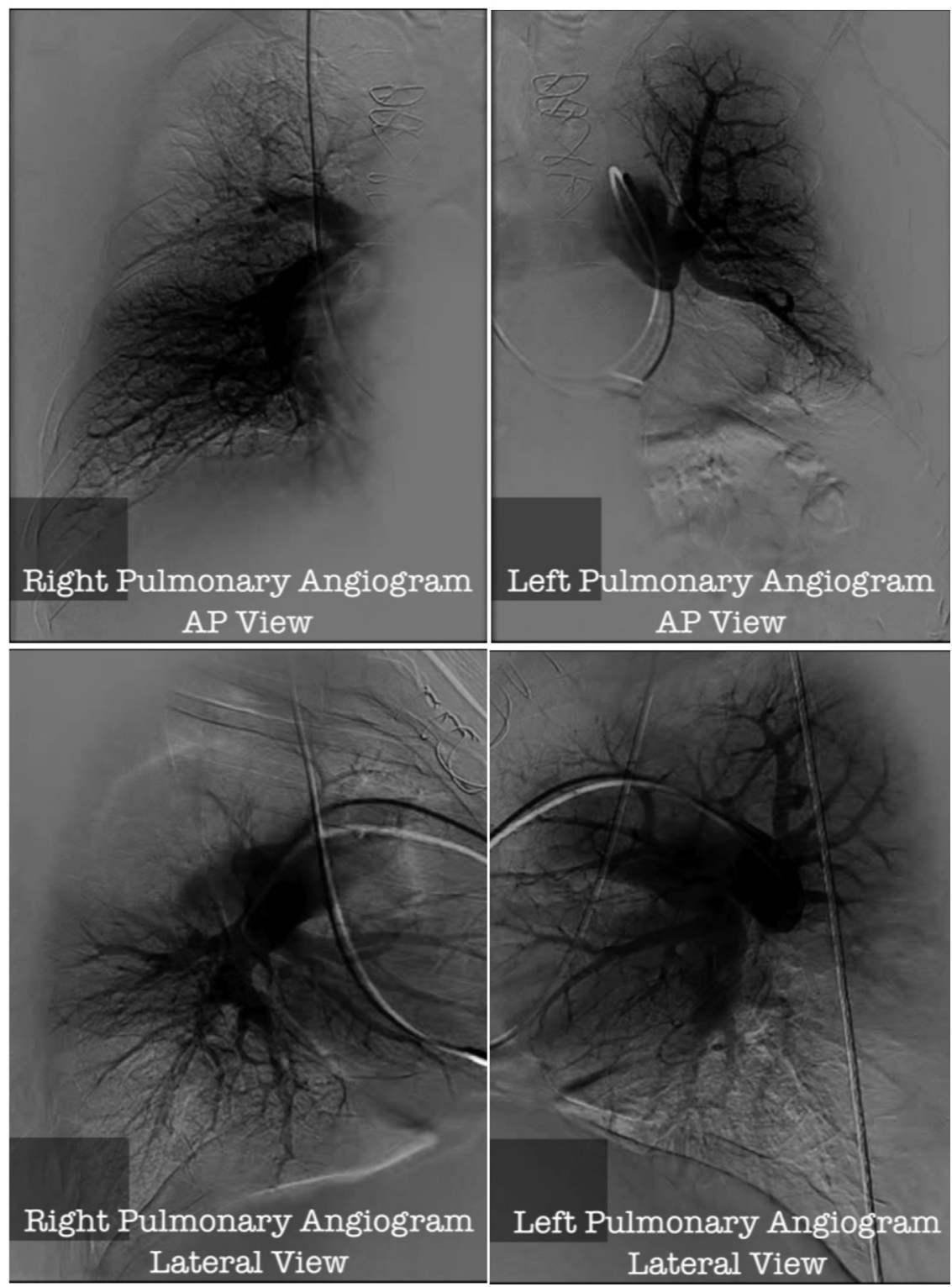

FIGURE 1. Selective preoperative pulmonary angiogram showing complete occlusion of left lower lobar pulmonary artery.

reduced, and a selective LLL angiogram performed. Vessel cut off was noted in the medial basilar branch of LLL PA, but no definite signs of extravasation were noted. Selective branch embolization of the same branch was performed using a 6-mm $\times 12-\mathrm{cm}$ nester coil. EBB was inflated again and ECMO flow was restored and patient was brought back to intensive care unit (Video 1, Part I).

This was not successful in achieving hemostasis, as noted in EBB deflation trial on the following day. Therefore, we proceeded with coil embolization more proximal to the previous area. Six AZUR (Terumo Interventional Systems, Somerset, NJ) detachable coils ranging from 4 to $10 \mathrm{~mm}$ in diameter were deployed into 3 basilar branches of the LLL PA and the main trunk of LLL PA (Figure 3 and Video 1,
Part II). This approach led to cessation of bleeding and allowed us to wean ECMO.

He was discharged to a rehabilitation facility on 37 th postoperative day. For modest residual PH, a single-agent PH therapy (riociguat) was started 4 months after the surgery. Repeat right heart catheterization 7 months after the surgery with riociguat showed mean PA pressure of $30 \mathrm{~mm} \mathrm{Hg}$, with pulmonary vascular resistance of 2.3 wood units.

\section{DISCUSSION}

The most life-threatening complication of PTE is acute pulmonary hemorrhage, ${ }^{1,2}$ and incidence is reported as high as $4 \%{ }^{1}$ It occurs when endarterectomy plane goes deep enough to perforate the PA, resulting in bleeding 


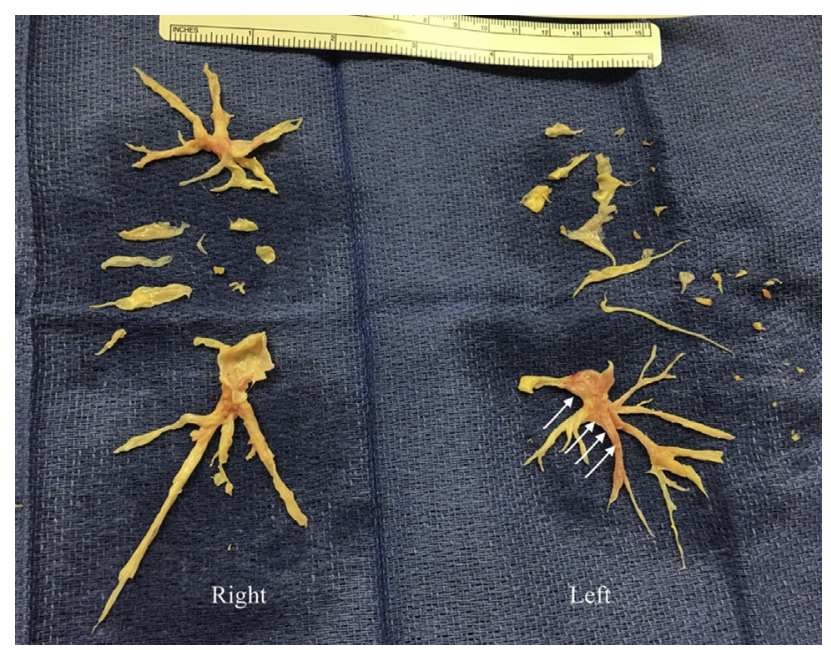

FIGURE 2. Thromboendarterectomy specimen. White arrows show severely adhered and partially calcified left lower lobe thrombus.

into surrounding airways. ${ }^{2,3}$ At our institution, we have observed 2 such cases in our first 246 PTEs $(0.8 \%)$.

In this case, the pacemaker lead as the origin of embolus lends itself to small-caliber thrombus material and more distal disease. ${ }^{4}$ Complete occlusion by calcified chronic thrombus material adherent distally presented a rare but important risk for bleeding.

VA-ECMO, bronchial blockers with or without fibrin glue, and reversal of anticoagulation is often successful in temporizing the bleeding. ${ }^{5}$ Selective PA coil embolization for persistent bleeding has not been reported to our knowledge. In our case, it was considered the only viable treatment option, given the distal nature of the PA injury, calcified or fragile vascular tissue, the presence of reperfusion lung injury, and the overall medical stability of the

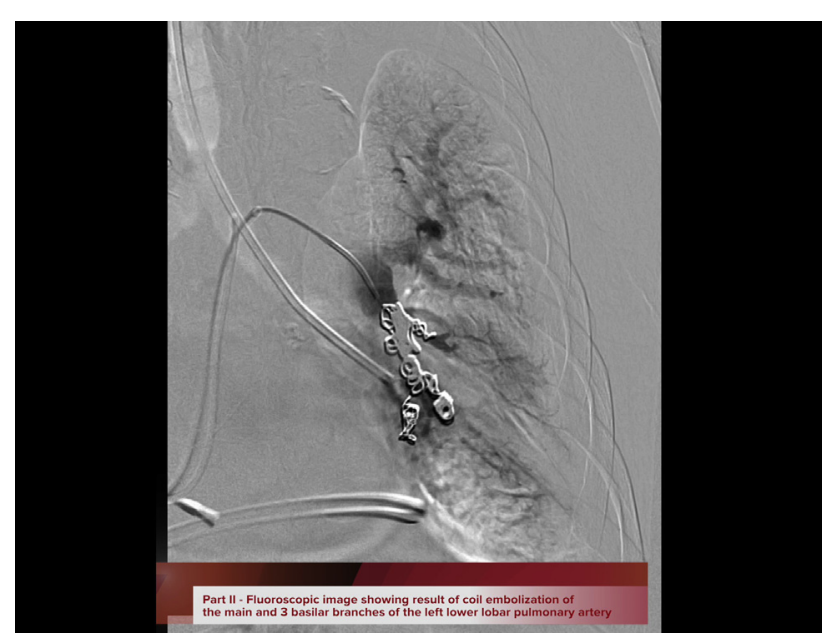

VIDEO 1. Part I: Fluoroscopic video showing result of left lower lobar medial basilar branch coil embolization. Part II: Fluoroscopic image showing result of coil embolization of the main and 3 basilar branches of the left lower lobar pulmonary artery. Video available at: https://www. jtcvs.org/article/S2666-2507(20)30429-6/fulltext.

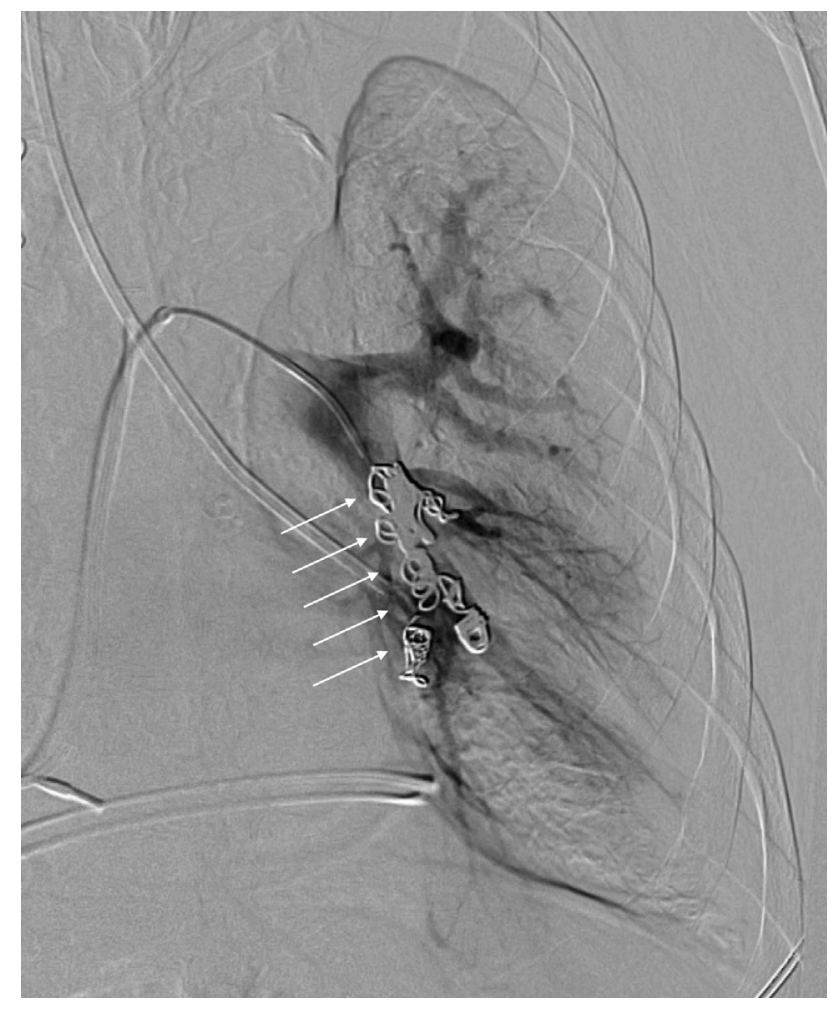

FIGURE 3. Fluoroscopic image showing coil embolization of the main and 3 basilar branches of the left lower lobe pulmonary artery. White arrows show coils.

patient. Although we deliberatively occluded the left lower lobar PA to control hemorrhage, we reasoned and anticipated that this would not have a major hemodynamic impact on right heart function, given excellent endarterectomy results in rest of pulmonary vasculature. Specifically, successful PTE in the right lung would provide marked pulmonary vascular surface area recruitment that would offset the loss of perfusion to the LLL. In keeping, the patient did not suffer any untoward hemodynamic consequences of LLL coil embolization. Informed consent was obtained from the patient regrading publication of pertinent data.

\section{References}

1. López Gude MJ, Pérez de la Sota E, Forteza Gil A, Centeno Rodríguez J, Eixerés A, Velázquez MT, et al. Pulmonary thromboendarterectomy in 106 patients with chronic thromboembolic pulmonary hypertension [in Spanish]. Arch Bronconeumol. 2015;51:502-8.

2. Madani MM, Auger WR, Pretorius V, Sakakibara N, Kerr KM, Kim NH, et al. Pulmonary endarterectomy: recent changes in a single institution's experience of more than 2,700 patients. Ann Thorac Surg. 2012;94:97-103.

3. Kolníková I, Kunstýř J, Lindner J, Lipš M, Kopecký P, Rulíšek J, et al. Extracorporeal membrane oxygenation used in a massive lung bleeding following pulmonary endarterectomy. Prague Med Rep. 2012;113:299-302.

4. Vaidya A, Gopalan D, Auger WR. Case 3: distal chronic thromboembolic dis ease-is surgery an option? In: Auger WR, ed. Clinical Cases in Chronic Thromboembolic Pulmonary Hypertension. New York: Springer; 2020:21-31.

5. Guth S, Wiedenroth CB, Wollenschläger M, Richter MJ, Ghofrani HA, Arlt M et al. Short-term venoarterial extracorporeal membrane oxygenation for massive endobronchial hemorrhage after pulmonary endarterectomy. J Thorac Cardiovasc Surg. 2018;155:643-9. 